\title{
Morphological Correlates of KIT and PDGFRA Genotypes in Gastrointestinal Stromal Tumour
}

\author{
Valli PRIYA (D, Niraj KUMARI D, Narendra KRISHNANI \\ Department of Pathology, Sanjay Gandhi Post Graduate Institute of Medical Sciences, LUCKNOW, INDIA
}

\begin{abstract}
Objective: The aim of the study was to study the clinicopathological and immunohistochemical features of gastrointestinal stromal tumours and correlation with KIT/PDGFRA mutations.

Material and Method: Eighty consecutive resected cases were genotyped for KIT exons 11, 9, 13, 17 and PDGFRA exons 18, 14, 12 and correlated with histomorphology by nonparametric tests.

Results: Forty-seven cases (58.8\%) were in the high-risk group. Males had higher rates of KIT exon 11 and PDGFRA exon 18 mutations than females ( $\mathrm{p}=0.03$ ). KIT and PDGFRA mutation frequencies were lower $(58.8 \%)$ than western data showing KIT exon 11 mutation in $63.8 \%$, KIT exon 9 mutation in 19\% and PDGFRA exon 18 mutation in 17\% of the cases. Extragastrointestinal stromal tumours (n=6) showed 100\% mutation. KIT exon 11 deletion was associated with gastric location $(60 \%)(\mathrm{p}=0.04)$, spindle cells $(63.3 \%)$, and high-risk stratification $(66.6 \%)$ $(\mathrm{p}=0.01)$ while KIT exon 9 mutation was common in small intestine $(66.7 \%)(\mathrm{p}=0.04)$, in higher risk groups $(66.7 \%)(\mathrm{p}=0.01)$ and $75 \%$ of codon 502-503 duplications ( $\mathrm{p}=0.03$ ). PDGFRA 18 mutation was common in males ( $\mathrm{p}=0.03$ ), in gastric location (62.5\%) ( $\mathrm{p}=0.04)$, in cases showing mild to moderate atypia (62.5\%) ( $\mathrm{p}=0.01)$ and lower risk stratification $(62.5 \%)(\mathrm{p}=0.01)$. KIT/PDGFRA mutations were significantly associated with gender $(\mathrm{p}=0.03)$, location $(\mathrm{p}=0.04)$, nuclear atypia $(\mathrm{p}=0.01)$ and risk stratification $(\mathrm{p}=0.01)$.
\end{abstract}

Conclusion: Morphological features and anatomic location may be useful in deciding molecular testing strategy, particularly in resource-limited settings, when a plethora of targetable mutations are present. An algorithm may be derived for genotyping with KIT exon 11 and PDGFRA exon 18 heading the list of targetable mutations. This approach may reduce financial burden on patients as well as workload on hospital staff.

Key Words: Gastrointestinal stromal tumours, Morphology, KIT, PDGFRA, Sequence analysis

\section{INTRODUCTION}

Gastrointestinal stromal tumour (GIST) is the most common mesenchymal neoplasms of the gastrointestinal tract (GIT) that arises from interstitial cells of Cajal (1). Morphologically, GISTs can be of spindle cell, epithelioid, or mixed phenotype that expresses CD117 (KIT) on immunohistochemistry. These tumours also express DOG1 and CD34 (2). Mutational analysis of GISTs have shown mutations in KIT mostly occurring in exon 11 followed by exon 9 and in PDGFRA exon 18 (3). KIT exon 11 mutations include deletions, point mutations and insertions. Certain morphological features are associated with KIT 11 deletions such as gastric location, spindle cell morphology and large tumour size $(4,5)$. KIT 11 insertions are also associated with spindle cell morphology but are generally seen in non-gastric locations (6). GISTs harbouring PDGFRA mutations are common in gastric, omental and mesenteric tumours showing nuclear pleomorphism, epithelioid morphology, plasmacytoid cells and multinucleated giant cells (7). Understanding the molecular and morphological correlations is important and may help to prioritize the sequential testing for GIST mutations, particularly in a resource-limited setting in developing countries. This study aims to compare the histomorphological and immunohistochemical features with various genotypes.

\section{MATERIALS and METHODS}

Eighty consecutive resected GISTs received within a period of 8 years were included in the study. Clinical findings and follow-up data were retrieved from the hospital records. All cases were reviewed for tumour size, location, histological type, cellularity, presence of atypia, necrosis, mitosis, secondary changes, lymph node and distant metastasis. Risk stratification was done according to Miettinen's classification (8). Extraintestinal GIST (EGIST) was defined by a combined approach of radiological, operative, gross and microscopic examination where the bulk of the tumour was outside the gastrointestinal tract and did not

(Turk Patoloji Derg 2020, 36:116-125)

Received : 18.05.2018 Accepted : 10.09.2019

Correspondence: Niraj KUMARI

Department of Pathology,

Sanjay Gandhi Post Graduate Institute of Medical Sciences,

LUCKNOW, INDIA

E-mail: nirajpath@gmail.com Phone: +800 4904562

Copyright $\odot 2020$ The Author(s). This is an open-access article published by Federation of Turkish Pathology Societies under the terms of the Creative Commons Attribution License which permits unrestricted use, distribution, and reproduction in any medium or format, provided the original work is properly cited. No use, distribution or reproduction is permitted which does not comply with these terms. 
show a clear cut connection with the bowel or stomach wall. Immunohistochemistry was performed for CD117 (DAKO, Denmark), DOG1 (Novocastra, United States), CD34 (DAKO, Denmark), SMA (DAKO, Denmark), S100 (DAKO, Denmark), desmin (DAKO, Denmark) and vimentin (DAKO, Denmark).

\section{Molecular Analysis}

DNA extraction was done from formalin-fixed paraffinembedded tissue using the QiaAmp FFPE kit. Polymerase chain reaction (PCR) was performed for KIT (exons 11, 9, 13,17 ) and PDGFRA (exons 18, 14, 12). Briefly, each PCR reaction was done in $25 \mu \mathrm{l}$ volume using $250 \mathrm{ng}$ of DNA on the ABI SimplyAmp ${ }^{\mathrm{TM}}$ thermal cycler followed by Sanger sequencing on the ABI 3130 Genetic Analyzer.

\section{Statistical Analysis}

Categorical variables were correlated using the Chi square test and Fisher's exact test where appropriate. Recurrencefree survival was analysed using Kaplan-Meier log-rank analysis. SPSS version 16 was used. A p value of $<0.05$ was considered significant. The study was approved by the institute's ethics committee.

\section{RESULTS}

\section{Demography}

Median age at presentation was 54 years (range $=16-80$ years) with a male to female ratio of $2: 1$. There were 38 gastric (47.5\%), 33 small intestinal (41\%), 3 colonic (4\%), 4 mesenteric (5\%) and 2 retroperitoneal (2.5\%) cases. In the small intestine, 10 cases were in the duodenum, 17 in the ileum and 6 in the jejunum. All patients were symptomatic at presentation with presence of abdominal mass, gastrointestinal bleeding or abdominal pain.

\section{Histopathology}

The majority of the gastric (89.5\%), small intestinal (90.9\%) and EGIST cases (83.3\%) showed moderate to high cellularity with colonic GISTs having conspicuously low cellularity $(66.7 \%),(p=0.15)$. The spindle cell type $(\mathrm{n}=52,65 \%)$ was predominantly present in all locations, followed by mixed $(n=20,25 \%)$ and epithelioid cell type $(\mathrm{n}=8,10 \%)$. Multinucleated tumour giant cells were seen scattered in 2 cases. The most common pattern was that of fascicles $(n=63,78.7 \%)$ followed by palisading (10 cases, $12.5 \%)$ and storiform (8 cases, $10 \%)(\mathrm{p}=0.15)$. Secondary changes included cystic degeneration and skenoid fibres in 11 cases each (13.7\%), of which $10(12.5 \%)$ were present in the small intestine, hyalinization ( 8 cases, $10 \%$ ), myxoid degeneration (7 cases, $8.7 \%$ ), paranuclear vacuolization (3 cases, $3.7 \%$ ), calcification (1 case, 1.2\%), congested vessels (4 cases, $5 \%$ ) and significant haemorrhage (6 case, $7.5 \%$ ).

The extent of tumour infiltration was limited to the submucosa in one case and muscularis propria in 9 cases. Exophytic GISTs $(n=15)$ involving the serosa alone were seen in 5 and serosa to muscularis propria in 10 cases. Twenty-nine cases (36.2\%) had entire thickness involvement with ulceration of overlying mucosa.

Overall CD117 and DOG-1 immunoreactivity were $93.8 \%$ $(n=75)$ and $91.3 \%$, respectively, with high concordance between the two markers; excluding five cases (6.25\%) with the CD117 (+)/DOG1(-); three (4\%) cases with CD117(-)/DOG1(+) and two cases (2.5\%) with CD117 $(-) / D O G 1(-)$ immune profile. The latter two cases were also KIT/PDGFRA wild type and the diagnosis was based on characteristic histomorphology, anatomical association with the gastrointestinal tract, presence of CD34 and absence of diffuse and strong expression of other immunohistochemical markers. The detailed gross, histological and immunohistochemical features are mentioned in Table I.

\section{Mutational Analysis}

Overall KIT and PDGFRA mutations were present in 47 cases $(58.8 \%)$.

KIT exon 11 mutations were seen in $30(63.8 \%)$ cases with simple deletions in 17 (56.7\%), point mutations in 10 (33.3\%) and complex mutations (deletions and duplication/ insertions) in 3 (10\%) cases. Codon 557-558 deletions (Figure 1A) were found in 15 cases (50\%).

KIT exon 9 mutations were found in 9 cases (19\%) which showed duplications Tyr502-503Asp in 4 cases and point mutations in 5 cases. These mutations were common in the small intestine (66.7\%) followed by EGIST (22.2\%) and stomach $(11.1 \%)$. The spectrum of mutations in KIT exon 11 and 9 are summarized in Table II.

PDGFRA exon 18 mutations were present in 8 cases (17\%), commonly located in the stomach $(62.5 \%)$, and had epithelioid/mixed morphology. D842V mutation was seen in 1 case whereas D842E was seen in 5 cases (Figure 1B). The spectrum of PDGFRA exon 18 mutations are summarised in Table III. No mutations were found in PDGFRA exons 12 and 14.

\section{Genotype and Clinicopathological Features}

Males had higher KIT exon 11 and PDGFRA mutations than females $(\mathrm{p}=0.03)$. GISTs with PDGFRA exon 18 mutations had smaller tumour size than cases with KIT 
exon 9 and exon 11 mutations ( $\mathrm{p}=0.01$ ). Nearly $67 \%$ of cases with KIT exon 11 mutations were present in the stomach that included 12 simple deletions, 7 point mutations and one complex mutation (Table III). Overall $66.7 \%$ of KIT exon 9 mutations were found in the small intestine ( $\mathrm{p}=0.04)$ of which codon 502-503 duplication was present in $75 \%$ of the small intestine ( $\mathrm{p}=0.03)$. PDGFRA exon 18 mutations were mostly present in the stomach
(62.5\%) and all D842 point mutations were found in the gastric location as well $(\mathrm{p}=0.04)$. All colonic GISTs were wild type whereas all EGISTs were mutant (KIT exon 11 deletion in 2 cases, KIT exon 11 point mutation in one case, KIT exon 9 duplication/point mutation and PDGFRA exon 18 point mutation in one case each). The correlation of the GIST genotype with clinicopathological variables and risk stratification is shown in Table IV.

Table I: Gross, histological and immunohistochemical features of GIST at different locations.

\begin{tabular}{|c|c|c|c|c|c|c|}
\hline \multicolumn{2}{|c|}{ Features } & Total $(\mathbf{n}=\mathbf{8 0})$ & $\begin{array}{c}\text { Stomach } \\
(\mathrm{n}=38)\end{array}$ & $\begin{array}{l}\text { Small intestine } \\
\qquad(\mathrm{n}=33)\end{array}$ & $\begin{array}{l}\text { Large intestine } \\
\qquad(\mathrm{n}=3)\end{array}$ & $\begin{array}{c}\text { EGIST } \\
(n=6)\end{array}$ \\
\hline \multicolumn{7}{|l|}{ Gross } \\
\hline \multicolumn{2}{|c|}{$\begin{array}{l}\text { Size }(\mathrm{cm}) \text { mean } \pm \text { Standard Deviation } \\
\text { (Minimum-Maximum) }\end{array}$} & $\begin{array}{c}10.3 \pm 6.28 \\
(1.5-30)\end{array}$ & $\begin{array}{l}9.8 \pm 5.1 \\
(1.5-21) \\
\end{array}$ & $\begin{array}{c}9.8 \pm 6.3 \\
(2-25)\end{array}$ & $\begin{array}{l}17 \pm 11.2 \\
(10.1-30)\end{array}$ & $\begin{array}{c}12.9 \pm 8.9 \\
(2.5-28)\end{array}$ \\
\hline \multicolumn{2}{|c|}{ Mucosal ulceration (n, \%) } & $38(475)$ & $18(47.4)$ & $19(57.6)$ & $1(33.3)$ & 0 \\
\hline \multicolumn{2}{|c|}{ Necrosis (n, \%) } & $19(23.7)$ & $11(29)$ & $5(15.1)$ & $1(33.3)$ & $2(33.6)$ \\
\hline \multicolumn{2}{|c|}{ Cystic degeneration (n, \%) } & $12(15)$ & $6(15.8)$ & $5(15.1)$ & $1(33.3)$ & 0 \\
\hline \multicolumn{7}{|c|}{ Microscopic } \\
\hline \multirow{3}{*}{ Cellularity } & Low, n (\%) & $10(12)$ & $4(10.5)$ & $3(9.1)$ & $2(66.7)$ & $1(16.7)$ \\
\hline & Moderate, $\mathrm{n}(\%)$ & $52(65)$ & $25(65.8)$ & $23(69.7)$ & $1(33.3)$ & $3(50)$ \\
\hline & High, n (\%) & $18(22.5)$ & $9(23.7)$ & $7(21.2)$ & 0 & $2(33.3)$ \\
\hline \multirow{3}{*}{ Cell type } & Spindle, n (\%) & $52(65)$ & $24(63.2)$ & $24(72.7)$ & $2(66.7)$ & $2(33.3)$ \\
\hline & Epithelioid, n (\%) & $8(10)$ & $5(13.2)$ & $1(3.1)$ & 0 & $1(16.7)$ \\
\hline & Mixed, n (\%) & $20(25)$ & $9(23.7)$ & $8(24.2)$ & $1(33.3)$ & $3(50)$ \\
\hline \multirow{4}{*}{ Pattern } & Fascicles, n (\%) & $63(78.7)$ & $31(38.7)$ & $30(37.5)$ & $1(33.3)$ & $2(33.3)$ \\
\hline & Palisading, $\mathrm{n}(\%)$ & $10(12.5)$ & $8(80)$ & $1(10)$ & 0 & $1(10)$ \\
\hline & Storiform, n (\%) & $8(10)$ & $3(40)$ & $5(60)$ & 0 & 0 \\
\hline & Sheets, $\mathrm{n}(\%)$ & $12(15)$ & $4(33.3)$ & $5(41.6)$ & $2(16.6)$ & $1(8.3)$ \\
\hline \multirow{2}{*}{ Mitosis } & $<5 / 50$ HPF, n (\%) & $40(50)$ & $18(47.4)$ & $20(60.6)$ & $1(33.3)$ & $1(16.7)$ \\
\hline & >5/50HPF, n (\%) & $40(50)$ & $20(52.6)$ & $13(39.4)$ & $2(66.7)$ & $5(83.3)$ \\
\hline \multirow{3}{*}{ Cellular atypia } & Mild, n (\%) & $37(46.3)$ & $16(42.1)$ & $17(51.5)$ & $1(33.3)$ & $2(33.3)$ \\
\hline & Moderate, $\mathrm{n}(\%)$ & $35(43.7)$ & $19(50)$ & $12(36.4)$ & $1(33.3)$ & $3(50)$ \\
\hline & Severe, n (\%) & $8(10)$ & $2(5.3)$ & $4(12.1)$ & $1(33.3)$ & $1(6.7)$ \\
\hline \multicolumn{2}{|c|}{ Lymph node metastasis } & $11(13.7)$ & $2(18.2)$ & $6(54.6)$ & $2(18.2)$ & $1(9.1)$ \\
\hline \multicolumn{2}{|c|}{ Distant metastasis } & $2(2.5)$ & $1(50)$ & 0 & 0 & $1(50)$ \\
\hline \multicolumn{2}{|c|}{ Lymph node metastasis } & $11(13.7)$ & $2(18.2)$ & $6(54.6)$ & $2(18.2)$ & $1(9.1)$ \\
\hline \multicolumn{7}{|c|}{ Immunohistochemistry } \\
\hline \multicolumn{2}{|c|}{ CD117 } & $75(93.8)$ & $36(94.7)$ & $30(84.8)$ & $3(100)$ & $6(100)$ \\
\hline \multicolumn{2}{|l|}{ DOG1 } & $73(91.3)$ & $38(97.4)$ & $28(84.8)$ & $3(100)$ & $4(66.7)$ \\
\hline \multicolumn{2}{|l|}{ CD34 } & $48(60)$ & $29(73.7)$ & $15(42.4)$ & $1(33.3)$ & $3(33.3)$ \\
\hline \multicolumn{2}{|l|}{ SMA } & $32(40)$ & $15(26.3)$ & $14(27.3)$ & 0 & $3(33.3)$ \\
\hline \multicolumn{2}{|l|}{ Desmin } & $13(16.2)$ & $6(15.8)$ & $3(6.1)$ & $1(33.3)$ & $3(33.3)$ \\
\hline \multicolumn{2}{|l|}{ S100 } & $29(36.2)$ & $17(36.8)$ & $9(24.2)$ & 0 & $3(16.7)$ \\
\hline
\end{tabular}


Table II: Spectrum of KIT mutations with location and immunohistochemistry.

\begin{tabular}{|c|c|c|c|c|c|c|c|c|c|c|c|}
\hline Age & Sex & Exon & Mutation & Locus & Location & CD117 & CD34 & SMA & S100 & Desmin & DOG1 \\
\hline 76 & $\mathrm{M}$ & 11 & Deletion & del 557-558 & Stomach & $\mathrm{P}$ & $\mathrm{N}$ & $\mathrm{P}$ & $\mathrm{N}$ & $\mathrm{N}$ & $\mathrm{P}$ \\
\hline 53 & $\mathrm{M}$ & 11 & Deletion & del 557-558 & Stomach & $\mathrm{P}$ & $\mathrm{P}$ & $\mathrm{P}$ & $\mathrm{N}$ & $\mathrm{N}$ & $\mathrm{P}$ \\
\hline 54 & $\mathrm{M}$ & 11 & Deletion & del 557-558 & Small bowel & $\mathrm{P}$ & $\mathrm{N}$ & $\mathrm{N}$ & $\mathrm{P}$ & $\mathrm{N}$ & $\mathrm{P}$ \\
\hline 56 & $\mathrm{M}$ & 11 & Deletion & del 557-558 & Stomach & $\mathrm{P}$ & $\mathrm{P}$ & $\mathrm{N}$ & $\mathrm{P}$ & $\mathrm{N}$ & $\mathrm{P}$ \\
\hline 60 & $\mathrm{M}$ & 11 & Deletion & del 557-559 & Extraintestinal & $\mathrm{P}$ & $\mathrm{P}$ & $\mathrm{N}$ & $\mathrm{N}$ & $\mathrm{N}$ & $\mathrm{P}$ \\
\hline 50 & $\mathrm{M}$ & 11 & Deletion & del 557-561 & Stomach & $\mathrm{P}$ & $\mathrm{N}$ & $\mathrm{N}$ & $\mathrm{N}$ & $\mathrm{N}$ & $\mathrm{P}$ \\
\hline 40 & $\mathrm{~F}$ & 11 & Deletion & del 557-559 & Stomach & $\mathrm{P}$ & $\mathrm{P}$ & $\mathrm{P}$ & $\mathrm{N}$ & $\mathrm{P}$ & $\mathrm{P}$ \\
\hline 62 & $\mathrm{M}$ & 11 & Deletion & del 557-558 & Stomach & $\mathrm{P}$ & $\mathrm{P}$ & $\mathrm{N}$ & $\mathrm{N}$ & $\mathrm{N}$ & $\mathrm{P}$ \\
\hline 47 & $\mathrm{M}$ & 11 & Deletion & del 547-558 & Stomach & $\mathrm{P}$ & $\mathrm{P}$ & $\mathrm{N}$ & $\mathrm{N}$ & $\mathrm{P}$ & $\mathrm{P}$ \\
\hline 57 & $\mathrm{~F}$ & 11 & Deletion & del 551-554 & Small bowel & $\mathrm{P}$ & $\mathrm{N}$ & $\mathrm{P}$ & $\mathrm{N}$ & $\mathrm{N}$ & $\mathrm{P}$ \\
\hline 43 & $\mathrm{M}$ & 11 & Deletion & del 556-564 & Small bowel & $\mathrm{P}$ & $\mathrm{N}$ & $\mathrm{P}$ & $\mathrm{N}$ & $\mathrm{N}$ & $\mathrm{P}$ \\
\hline 73 & $\mathrm{M}$ & 11 & Deletion & del 552-557 & Stomach & $\mathrm{P}$ & $\mathrm{P}$ & $\mathrm{P}$ & $\mathrm{N}$ & $\mathrm{N}$ & $\mathrm{P}$ \\
\hline 51 & $\mathrm{M}$ & 11 & Deletion & del 553-556 & Stomach & $\mathrm{P}$ & $\mathrm{P}$ & $\mathrm{N}$ & $\mathrm{N}$ & $\mathrm{N}$ & $\mathrm{P}$ \\
\hline 58 & $\mathrm{M}$ & 11 & Deletion & del 557-560 & Stomach & $\mathrm{P}$ & $\mathrm{P}$ & $\mathrm{N}$ & $\mathrm{P}$ & $\mathrm{N}$ & $\mathrm{P}$ \\
\hline 35 & $\mathrm{M}$ & 11 & Deletion & del 560 & Small bowel & $\mathrm{P}$ & $\mathrm{N}$ & $\mathrm{N}$ & $\mathrm{N}$ & $\mathrm{N}$ & $\mathrm{P}$ \\
\hline 61 & $\mathrm{M}$ & 11 & Deletion & del 554-557 & Small bowel & $\mathrm{P}$ & $\mathrm{N}$ & $\mathrm{N}$ & $\mathrm{N}$ & $\mathrm{N}$ & $\mathrm{P}$ \\
\hline 45 & $\mathrm{M}$ & 11 & $\mathrm{PM}$ & PT val560asp & Small bowel & $\mathrm{P}$ & $\mathrm{N}$ & $\mathrm{P}$ & $\mathrm{P}$ & $\mathrm{N}$ & $\mathrm{P}$ \\
\hline 57 & $\mathrm{M}$ & 11 & $\mathrm{PM}$ & PT ser590aspgn & Small bowel & $\mathrm{P}$ & $\mathrm{N}$ & $\mathrm{N}$ & $\mathrm{N}$ & $\mathrm{N}$ & $\mathrm{N}$ \\
\hline 54 & $\mathrm{M}$ & 11 & $\mathrm{PM}$ & PT arg588lys & Small bowel & $\mathrm{P}$ & $\mathrm{P}$ & $\mathrm{P}$ & $\mathrm{P}$ & $\mathrm{N}$ & $\mathrm{P}$ \\
\hline 55 & $\mathrm{~F}$ & 11 & $\mathrm{PM}$ & PT leu 576 pro & Extraintestinal & $\mathrm{P}$ & $\mathrm{P}$ & $\mathrm{P}$ & $\mathrm{N}$ & $\mathrm{P}$ & $\mathrm{P}$ \\
\hline 42 & $\mathrm{M}$ & 11 & $\mathrm{PM}$ & PT try 557 gly & Stomach & $\mathrm{P}$ & $\mathrm{P}$ & $\mathrm{P}$ & $\mathrm{P}$ & $\mathrm{N}$ & $\mathrm{P}$ \\
\hline 47 & $\mathrm{M}$ & 11 & $\mathrm{PM}$ & PT val 559 asp & Stomach & $\mathrm{P}$ & $\mathrm{N}$ & $\mathrm{N}$ & $\mathrm{P}$ & $\mathrm{N}$ & $\mathrm{P}$ \\
\hline 47 & $\mathrm{M}$ & 11 & $\mathrm{PM}$ & PT val 559 gly & Stomach & $\mathrm{P}$ & $\mathrm{P}$ & $\mathrm{N}$ & $\mathrm{N}$ & $\mathrm{N}$ & $\mathrm{P}$ \\
\hline 67 & $\mathrm{M}$ & 11 & $\mathrm{PM}$ & PT gly559pro & Stomach & $\mathrm{P}$ & $\mathrm{P}$ & $\mathrm{N}$ & $\mathrm{P}$ & $\mathrm{N}$ & $\mathrm{P}$ \\
\hline 49 & $\mathrm{M}$ & 11 & $\mathrm{PM}$ & PT tyr553asn & Stomach & $\mathrm{P}$ & $\mathrm{P}$ & $\mathrm{P}$ & $\mathrm{P}$ & $\mathrm{N}$ & $\mathrm{P}$ \\
\hline 60 & $\mathrm{~F}$ & 11 & $\mathrm{PM}$ & PT tyr553asn & Stomach & $\mathrm{P}$ & $\mathrm{P}$ & $\mathrm{N}$ & $\mathrm{N}$ & $\mathrm{N}$ & $\mathrm{P}$ \\
\hline 69 & M & 11 & Complex & $\begin{array}{c}\text { complex del-ins } \\
557-561 \text {, ins 557- } \\
558 \\
\end{array}$ & Stomach & $\mathrm{P}$ & $\mathrm{P}$ & $\mathrm{N}$ & $\mathrm{N}$ & $\mathrm{N}$ & $\mathrm{P}$ \\
\hline 71 & M & 11 & Complex & $\begin{array}{c}\text { complex del-subs } \\
557+\text { one } 556\end{array}$ & Small bowel & $\mathrm{P}$ & $\mathrm{P}$ & $\mathrm{N}$ & $\mathrm{N}$ & $\mathrm{N}$ & $\mathrm{P}$ \\
\hline 57 & M & 11 & Complex & $\begin{array}{c}\text { complex ins } 575- \\
576\end{array}$ & Extraintestinal & $\mathrm{P}$ & $\mathrm{N}$ & $\mathrm{P}$ & $\mathrm{P}$ & $\mathrm{N}$ & $\mathrm{P}$ \\
\hline 40 & M & 11 & Complex & $\begin{array}{c}\text { del homozygous } \\
554-561\end{array}$ & Stomach & $\mathrm{P}$ & $\mathrm{P}$ & $\mathrm{N}$ & $\mathrm{N}$ & $\mathrm{N}$ & $\mathrm{P}$ \\
\hline 49 & $\mathrm{M}$ & 9 & Duplication & dup 502-503 & Small bowel & $\mathrm{P}$ & $\mathrm{P}$ & $\mathrm{N}$ & $\mathrm{P}$ & $\mathrm{N}$ & $\mathrm{P}$ \\
\hline 54 & $\mathrm{M}$ & 9 & Duplication & dup 502-503 & Extraintestinal & $\mathrm{P}$ & $\mathrm{N}$ & $\mathrm{N}$ & $\mathrm{P}$ & $\mathrm{P}$ & $\mathrm{P}$ \\
\hline 53 & $\mathrm{M}$ & 9 & Duplication & dup 502-503 & Small bowel & $\mathrm{P}$ & $\mathrm{P}$ & $\mathrm{P}$ & $\mathrm{N}$ & $\mathrm{N}$ & $\mathrm{P}$ \\
\hline 68 & $\mathrm{M}$ & 9 & Duplication & dup 502-503 & Small bowel & $\mathrm{P}$ & $\mathrm{P}$ & $\mathrm{P}$ & $\mathrm{P}$ & $\mathrm{N}$ & $\mathrm{P}$ \\
\hline 46 & $\mathrm{~F}$ & 9 & $\mathrm{PM}$ & PT leu472isoleu & Small bowel & $\mathrm{P}$ & $\mathrm{P}$ & $\mathrm{P}$ & $\mathrm{N}$ & $\mathrm{N}$ & $\mathrm{P}$ \\
\hline 65 & $\mathrm{~F}$ & 9 & $\mathrm{PM}$ & PT phe504ser & Extraintestinal & $\mathrm{P}$ & $\mathrm{N}$ & $\mathrm{P}$ & $\mathrm{P}$ & $\mathrm{P}$ & $\mathrm{N}$ \\
\hline 38 & $\mathrm{M}$ & 9 & $\mathrm{PM}$ & PT thr488meth & Small bowel & $\mathrm{P}$ & $\mathrm{P}$ & $\mathrm{P}$ & $\mathrm{P}$ & $\mathrm{P}$ & $\mathrm{N}$ \\
\hline 77 & $\mathrm{M}$ & 9 & $\mathrm{PM}$ & PT cys491gly & Stomach & $\mathrm{P}$ & $\mathrm{P}$ & $\mathrm{N}$ & $\mathrm{N}$ & $\mathrm{N}$ & $\mathrm{P}$ \\
\hline 34 & $\mathrm{~F}$ & 9 & $\mathrm{PM}$ & PT thr488alan & Small bowel & $\mathrm{P}$ & $\mathrm{P}$ & $\mathrm{N}$ & $\mathrm{N}$ & $\mathrm{N}$ & $\mathrm{P}$ \\
\hline
\end{tabular}

${ }^{\star}$ PM: Point mutation, P: Positive, N: Negative. 


\section{Genotype and Histomorphology}

KIT exon 11 and 9 mutations were predominantly associated with spindle cell morphology (Figure 2A) compared to PDGFRA mutations that had epithelioid (Figure 2B) or mixed phenotype $(\mathrm{p}=0.4)$. Mild to moderate nuclear atypia was significantly associated with KIT exon $11(100 \%)$ and exon 9 mutations (100\%) whereas $37.5 \%$ cases with PDGFRA exon 18 mutation and $15 \%$ of wild type GISTs showed severe atypia including plasmacytoid cells and binucleate tumour giant cells ( $\mathrm{p}=0.01$ ) (Figure 2C).

A diffuse sheet-like pattern was seen in 10 cases; 6 of which harbored KIT exon 11 deletions and 4 were wild type. All cases with substitutions and insertions had fascicles and/ or a palisaded pattern. Paranuclear vacuolization was commonly observed in cases with KIT exon 9 mutations (Figure 2D). It may be assumed that cases with both KIT exon11 deletions and wild type have an aggressive course with high cellularity and hence have a diffuse sheet-like pattern.

\section{Genotype and Survival}

Follow-up was available in 73 patients with median followup of 27 and mean follow-up of 34.2 months \pm 26.7 (range: 1-101 months). Recurrence was observed in 12 patients (9 males, 3 females) within 6 to 34 months post-surgery in 9 high risk, 2 intermediate risk and 1 low malignant risk cases. Six recurrent cases (50\%) showed KIT exon 11 mutation with 4 cases (33.4\%) having codon 557-558 deletion and two (16.7\%) having point mutations. Median recurrence free survival (RFS) in patients with wild type GIST was higher than in patients with mutated GIST (79 months vs. 67 months, respectively; $\mathrm{p}=0.9$ ) (Figure 3A). The 5-year RFS in patients with KIT and PDGFRA wild type was also higher ( $87 \%$ vs.48\%) than in mutant GIST $(\mathrm{p}=0.6)$. The median RFS in patients with codon 557-558 deletion was 67 months compared to 84 months in patients with all other missense mutations $(\mathrm{p}=0.6)$ (Figure 3B). The 5-year RFS was $42 \%$ in patients with codon 557-558 deletion and $53 \%$ in other missense mutations $(\mathrm{p}=0.7)$ of

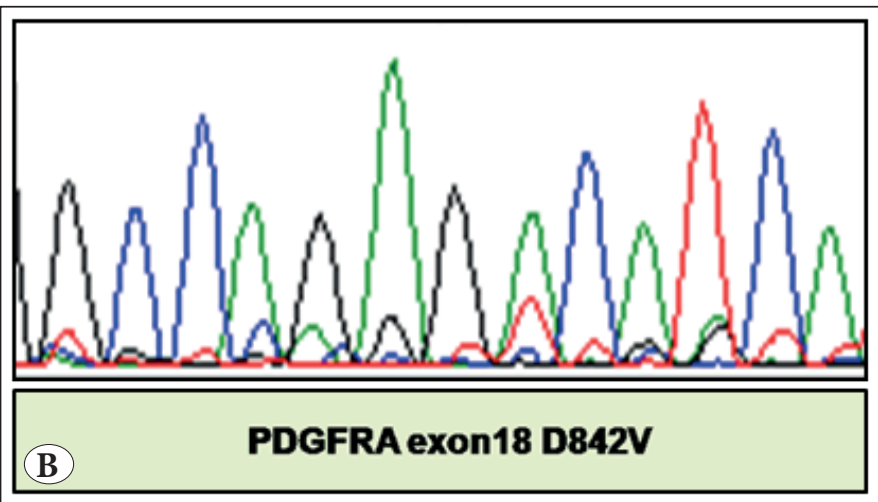

Figure 1: A) Electrophoretogram displays KIT exon 11 deletion in codons 557, 558. B) Electrophoretogram displays point mutation in codon 842 of PDGFRA exon 18.

Table III: Spectrum of PDGFRA exon 18 mutations with locations and immunohistochemistry.

\begin{tabular}{|c|c|c|c|c|c|c|c|c|c|c|}
\hline Age & Sex & Mutation & Locus & Location & CD117 & CD34 & SMA & S100 & Desmin & DOG1 \\
\hline 60 & $\mathrm{M}$ & PM & PT aspt842glut & Stomach & $\mathrm{P}$ & $\mathrm{N}$ & $\mathrm{N}$ & $\mathrm{N}$ & $\mathrm{N}$ & $\mathrm{P}$ \\
\hline 39 & $\mathrm{M}$ & $\mathrm{PM}$ & PT aspt842glut & Extraintestinal & $\mathrm{P}$ & $\mathrm{P}$ & $\mathrm{N}$ & $\mathrm{N}$ & $\mathrm{N}$ & $\mathrm{N}$ \\
\hline 53 & $\mathrm{M}$ & $\mathrm{PM}$ & PT aspt842glut & Stomach & $\mathrm{P}$ & $\mathrm{P}$ & $\mathrm{P}$ & $\mathrm{P}$ & $\mathrm{N}$ & $\mathrm{P}$ \\
\hline 75 & $\mathrm{M}$ & $\mathrm{PM}$ & PT aspt842glut & Stomach & $\mathrm{N}$ & $\mathrm{P}$ & $\mathrm{N}$ & $\mathrm{P}$ & $\mathrm{N}$ & $\mathrm{P}$ \\
\hline 47 & M & $\mathrm{PM}$ & PT aspt842val & Stomach & $\mathrm{P}$ & $\mathrm{P}$ & $\mathrm{P}$ & $\mathrm{P}$ & $\mathrm{P}$ & $\mathrm{P}$ \\
\hline 80 & $\mathrm{M}$ & $\mathrm{PM}$ & PT aspt842glut & Stomach & $\mathrm{P}$ & $\mathrm{P}$ & $\mathrm{P}$ & $\mathrm{N}$ & $\mathrm{P}$ & $\mathrm{P}$ \\
\hline 43 & M & PM & PT his817pro & Small bowel & $\mathrm{P}$ & $\mathrm{N}$ & $\mathrm{N}$ & $\mathrm{N}$ & $\mathrm{N}$ & $\mathrm{P}$ \\
\hline 34 & M & $\mathrm{PM}$ & PT Leu826Glu & Small bowel & $\mathrm{P}$ & $\mathrm{N}$ & $\mathrm{N}$ & $\mathrm{N}$ & $\mathrm{N}$ & $\mathrm{P}$ \\
\hline 60 & M & $\mathrm{PM}$ & PT aspt842glut & Stomach & $\mathrm{P}$ & $\mathrm{N}$ & $\mathrm{N}$ & $\mathrm{N}$ & $\mathrm{N}$ & $\mathrm{P}$ \\
\hline
\end{tabular}

${ }^{\star}$ PM: Point mutation, P: Positive, N: Negative. 
KIT 11. The codon 557-558 deletion also showed decreased median RFS of 68 months (5-year survival: $42 \%$ ) compared to wild type GISTs with RFS of 79 months (5-year survival: 92\%) $(\mathrm{p}=0.4)$ (Figure 3C).

\section{DISCUSSION}

GISTs are mesenchymal tumours with malignant risk potential ranging from very low to high risk. The diagnosis of GIST relies on a panel of immunohistochemical markers whereas genotyping of GIST is essential for dosage and predicting the response of tyrosine kinase inhibitors. Since GISTs harbour various mutations having a wide range of frequency with their available targets, it is prudent to prioritize genetic mutation in this disease and use the resources judiciously, especially in developing countries.

In the present study, tumour size ranged from 1.5-30 $\mathrm{cm}$ with a mean of $10.3 \mathrm{~cm}$, with larger tumours in large intestinal (mean: $17 \mathrm{~cm}$ ) and extraintestinal location (mean:
$12.9 \mathrm{~cm})$ compared to the stomach and small intestine (mean-9.8 cm). In the series by Miettinen et al., the median size of gastric GISTs was $6 \mathrm{~cm}$ (9) and several European series have reported a median size of 5-5.6 $\mathrm{cm}(2-10 \mathrm{~cm})$ $(10,11)$. On the contrary, median tumour size from other Indian series varies from 10.6 to $10.9 \mathrm{~cm}(12,13)$, similar to our series which could be related to later admission to the hospital at an advanced symptomatic stage.

Mixed and epithelioid cell types are usually encountered in a gastric location (14). Interestingly, in the present study $50 \%$ of our EGISTs also showed a mixed cell type and this has not been observed in the EGIST of other series $(9,10)$.

High concordance (87.5\%) was observed between CD117 and DOG1 immunoreactivity; however, four of five (80\%) CD117 negative cases stained for DOG1. Foo et al. have reported one third of CD117 negative cases to be positive for DOG1 (15).
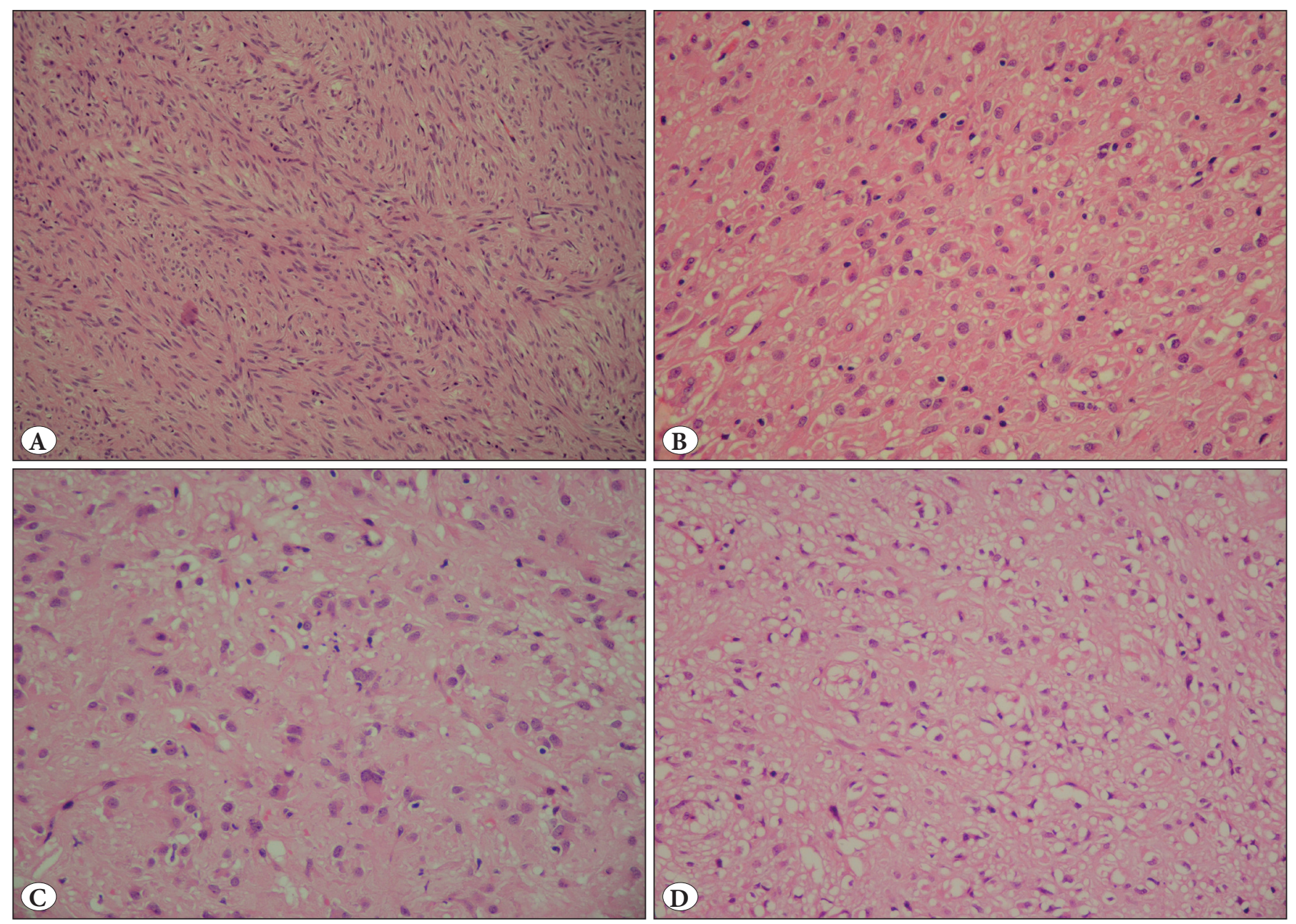

Figure 2: A) Spindled tumour cells arranged in fascicles in a KIT11 mutant GIST (H\&E; x20). B) Tumour cells with epithelioid morphology, round nuclei, vesicular chromatin and abundant eosinophilic cytoplasm (PDGFRA mutant gastric GIST) (H\&E; x20). C) Tumour cells with moderate cellular pleomorphism in PDGFRA mutant EGIST (H\&E; x20). D) Tumour cells with paranuclear vacuolation in a KIT exon 9 mutant small intestinal GIST (H\&E; x20) 
KIT/PDGFRA mutation frequency was lower in the present study compared to western data. This may be explained by ethnical variation which is well known for lung and colorectal cancers (16). Although genotyping studies from India and other Asian countries are fewer, the series from Taiwan and China also showed similar lower rate of mutations in KIT and PDGFRA genes (17-19). In our cases, KIT exon 11 and PDGFRA exon 18 mutations were commonly seen in the stomach whereas KIT exon 9 mutations were seen in the small intestine, similar to other studies $(20,21)$. However, Daniel et al. showed KIT 11 deletions to have different locations having a wide spectrum of morphology (22). A peculiar finding noted in this study was the presence of $100 \%$ mutation in EGISTs.
Male gender had higher rates of both KIT11 and PDGFRA mutations as well as wild genotype as compared to females ( $\mathrm{p}=0.03$ ), indicating that all kinds of GIST genotype is possible in males (20). Lv et al. showed that all mutations were more common and associated with poor RFS in males (23). PDGFRA mutation showed a significant correlation with male gender in the present study ( $\mathrm{p}=0.02$ ), similar to Daniels et al.; however, KIT 9 mutation was equally present among both sexes (22). PDGFRA exon 18 mutations had smaller tumour size than cases with KIT exon 9 and 11 mutations $(\mathrm{p}=0.01)$, similar to the series of Wozniak et al. (20).

Table IV: Correlation of histopathological variables with mutation.

\begin{tabular}{|c|c|c|c|c|c|c|}
\hline \multicolumn{2}{|c|}{ Variables } & $\begin{array}{c}\text { KIT exon } 11 \\
(n=30)(\%)\end{array}$ & $\begin{array}{c}\text { KIT exon } 9 \\
(n=9)(\%)\end{array}$ & $\begin{array}{c}\text { PDGFRA exon } 18 \\
(n=8)(\%)\end{array}$ & $\begin{array}{l}\text { Wild type } \\
(\mathrm{n}=33)(\%)\end{array}$ & p- value ${ }^{\star}$ \\
\hline \multirow{2}{*}{ Gender } & Male & $26(86.6)$ & $6(66.6)$ & $8(100)$ & $20(60.6)$ & \multirow{2}{*}{0.03} \\
\hline & Female & $4(13.4)$ & $3(33.4)$ & 0 & $13(39.4)$ & \\
\hline \multirow{4}{*}{ Location } & Stomach & $18(60)$ & $1(11.1)$ & $5(62.5)$ & $14(42.4)$ & \multirow{4}{*}{0.04} \\
\hline & Small intestine & $9(30)$ & $6(66.7)$ & $2(25)$ & $16(48.4)$ & \\
\hline & Colon & 0 & 0 & 0 & $3(9.2)$ & \\
\hline & Extraintestinal & $3(10)$ & $2(22.2)$ & $1(12.5)$ & 0 & \\
\hline \multirow{4}{*}{ Tumour size } & $0-2 \mathrm{~cm}$ & 0 & 0 & 0 & $2(6.1)$ & \multirow{4}{*}{0.1} \\
\hline & $2.1-5 \mathrm{~cm}$ & $5(16.7)$ & $4(44.5)$ & $3(37.5)$ & $3(9.2)$ & \\
\hline & $5.1-10 \mathrm{~cm}$ & $12(40)$ & $2(22.2)$ & $4(50)$ & $11(33.3)$ & \\
\hline & $>10 \mathrm{~cm}$ & $13(43.3)$ & $3(33.3)$ & $1(12.5)$ & $17(51.5)$ & \\
\hline \multirow{3}{*}{ Cell type } & Spindle & $19(63.3)$ & $7(77.8)$ & $3(37.5)$ & $23(69.7)$ & \multirow{3}{*}{0.4} \\
\hline & Epithelioid & $2(6.7)$ & 0 & $2(25)$ & $3(9.2)$ & \\
\hline & Mixed & $9(30)$ & $2(22.2)$ & $3(37.5)$ & $7(21.2)$ & \\
\hline \multirow{3}{*}{$\begin{array}{l}\text { Cellular } \\
\text { atypia }\end{array}$} & Mild & $18(60)$ & $4(44.5)$ & $3(37.5)$ & $12(36.3)$ & \multirow{3}{*}{0.01} \\
\hline & Moderate & $12(40)$ & $5(55.5)$ & $2(25)$ & $16(48.4)$ & \\
\hline & Severe & 0 & 0 & $3(37.5)$ & $5(15.3)$ & \\
\hline \multirow{2}{*}{ Mitosis group } & $<5 / 50 \mathrm{HPF}$ & $11(36.7)$ & $5(55.5)$ & $6(75)$ & $18(54.6)$ & \multirow{2}{*}{0.2} \\
\hline & $>5 / 50 \mathrm{HPF}$ & $19(63.3)$ & $4(44.5)$ & $2(25)$ & $15(45.4)$ & \\
\hline \multirow[t]{5}{*}{ Risk group } & None & 0 & 0 & 0 & $1(3)$ & 0.01 \\
\hline & Very low & $1(3.3)$ & 0 & $3(37.5)$ & 0 & \\
\hline & Low & $4(13.3)$ & $3(33.3)$ & $1(12.5)$ & $6(18.2)$ & \\
\hline & Intermediate & $5(16.6)$ & 0 & $1(12.5)$ & $8(24.2)$ & \\
\hline & High & $20(66.6)$ & $6(66.7)$ & $3(37.5)$ & $18(54.5)$ & \\
\hline \multicolumn{2}{|c|}{ Lymph node metastasis } & $2(6.7)$ & $1(11.1)$ & $1(12.5)$ & $7(21.2)$ & 0.4 \\
\hline \multicolumn{2}{|c|}{ Distant metastasis } & $2(6.7)$ & 0 & 0 & 0 & 0.3 \\
\hline \multicolumn{2}{|l|}{ Recurrence } & $6(20)$ & 0 & $2(40)$ & $4(12.1)$ & 0.3 \\
\hline
\end{tabular}

${ }^{\star}$ Pearson Chi square and Fisher Exact tests were used wherever applicable 
Median recurrence free survival in all mutant and wild GIST

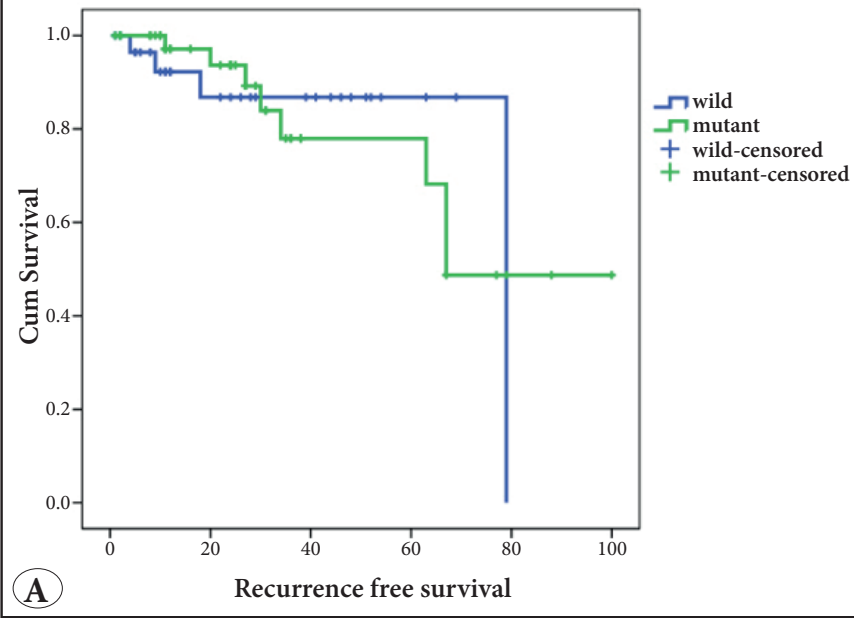

Median recurrence free survival in KIT exon 11 codon 557-558 deletion and all other missense mutations

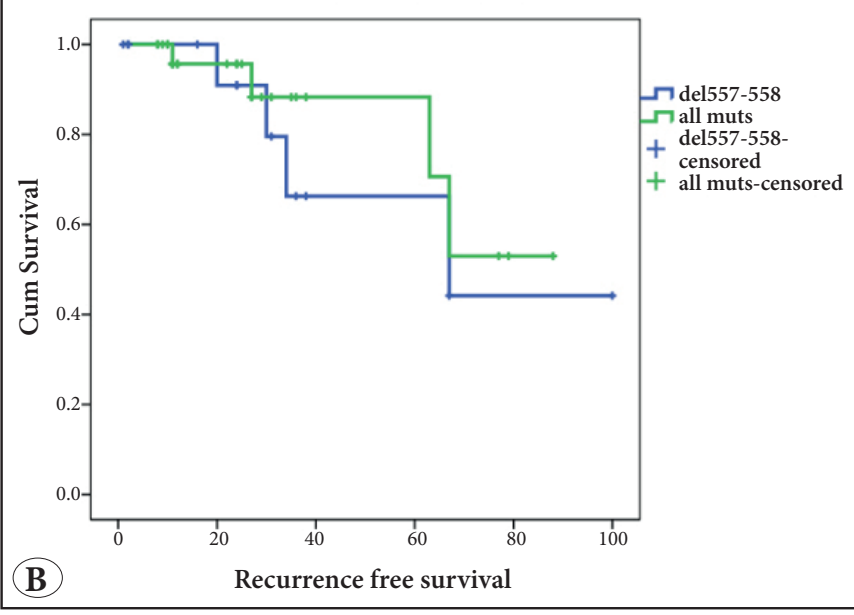

Median recurrence free survival in KIT exon 11 codon 557-558 deletions and wild type GISTs

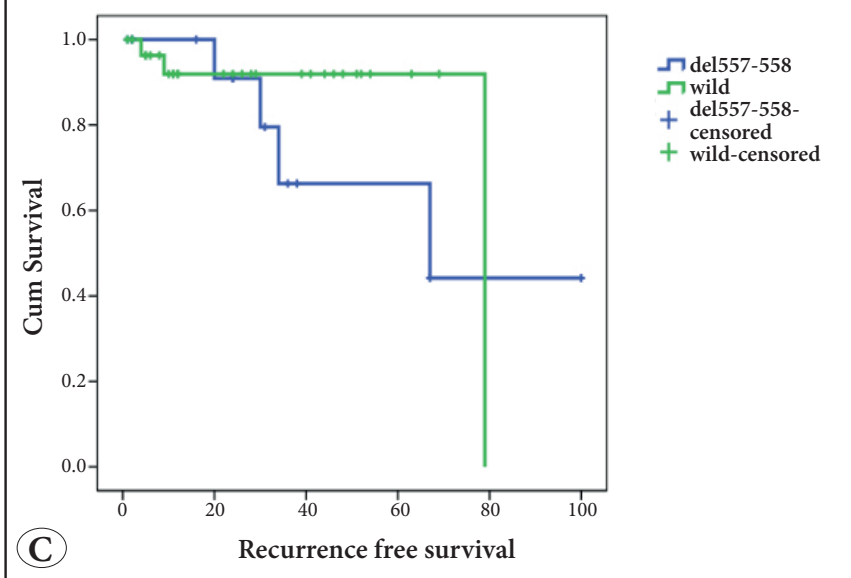

Figure 3: Survival curves: A) Wild and mutant GISTs. B) KIT exon 11 codon 557-558 deletion and all other mutant GISTs. C) KIT exon 11 codon 557-558 deletion and wild GISTs.
Agaimy et al. and Daum et al. noted that epithelioid or mixed cell morphology, presence of mast cells, multinucleated giant cells and myxoid stroma were more frequently associated with PDGFRA mutation $(7,24)$. We also observed similar findings where 5 of 8 PDGFRA mutated cases had epithelioid morphology and two cases had plasmacytoid cells and multinucleated cells. Unfortunately, SDH, KRAS and BRAF mutations could not be performed in KIT-PDGFRA wild type cases due to financial constraints, which is one of the major limitations of this study.

Morphological predictors of prognosis or RFS were mitotic activity $(\mathrm{p}=0.009)$, tumour necrosis $(\mathrm{p}=0.04)$, and cellular atypia $(\mathrm{p}=0.03)$.

\section{Suggested Algorithm for Molecular Testing in GIST Based on Morphological Features}

Considering all histomorphological or genotypical features observed in the present study, we can argue that certain morphological features can be associated with a particular genotype. Therefore, we can suggest that the sequence of testing can be modified especially for patients/centres where financial affordability for multiple investigations is a concern (Figure 4). As most of the GIST mutations are mutually exclusive, the order of mutation testing can be devised for each set of morphological features. Tumours in the gastric location with spindle cell morphology and high mitosis may be tested for KIT exon 11 while gastric tumours with low mitosis, epithelioid morphology and low cellular atypia may be tested initially for PDGFRA exon 18 in the first phase, or if feasible, gastric GISTs should be first tested for KIT exon 11 followed by PDGFRA exon 18. If both are wild type then one may proceed with KIT exon 9 testing. About $20-40 \%$ of double negative GISTs are positive for SDH mutations and another $15 \%$ of triple negative (KIT, PDGFRA, SDH negative) cases harbour mutations in $\operatorname{RAS} / \operatorname{BRAF}(25,26)$, which may be checked if the above mutations are negative. SDH immunohistochemistry can be done either following or in conjunction with KIT exon 11 and 9 mutation testing. Later, the BRAF, KIT exons 13, 17 and PDGFRA exon 12 mutations may be tested, which account for $<5 \%$ of all mutations. Most SDH deficient GISTs have characteristic dumb bell/lobulated shape with thick fibrous bands, epithelioid morphology and frequent lymph node metastasis; SDH testing may precede KIT exon 11 mutation testing for suspected cases. It should be noted that this suggested algorithm is suited mostly for resource limited situations, and does not disagree with studying multiple mutations if laboratory resources and the patient's status permits. 


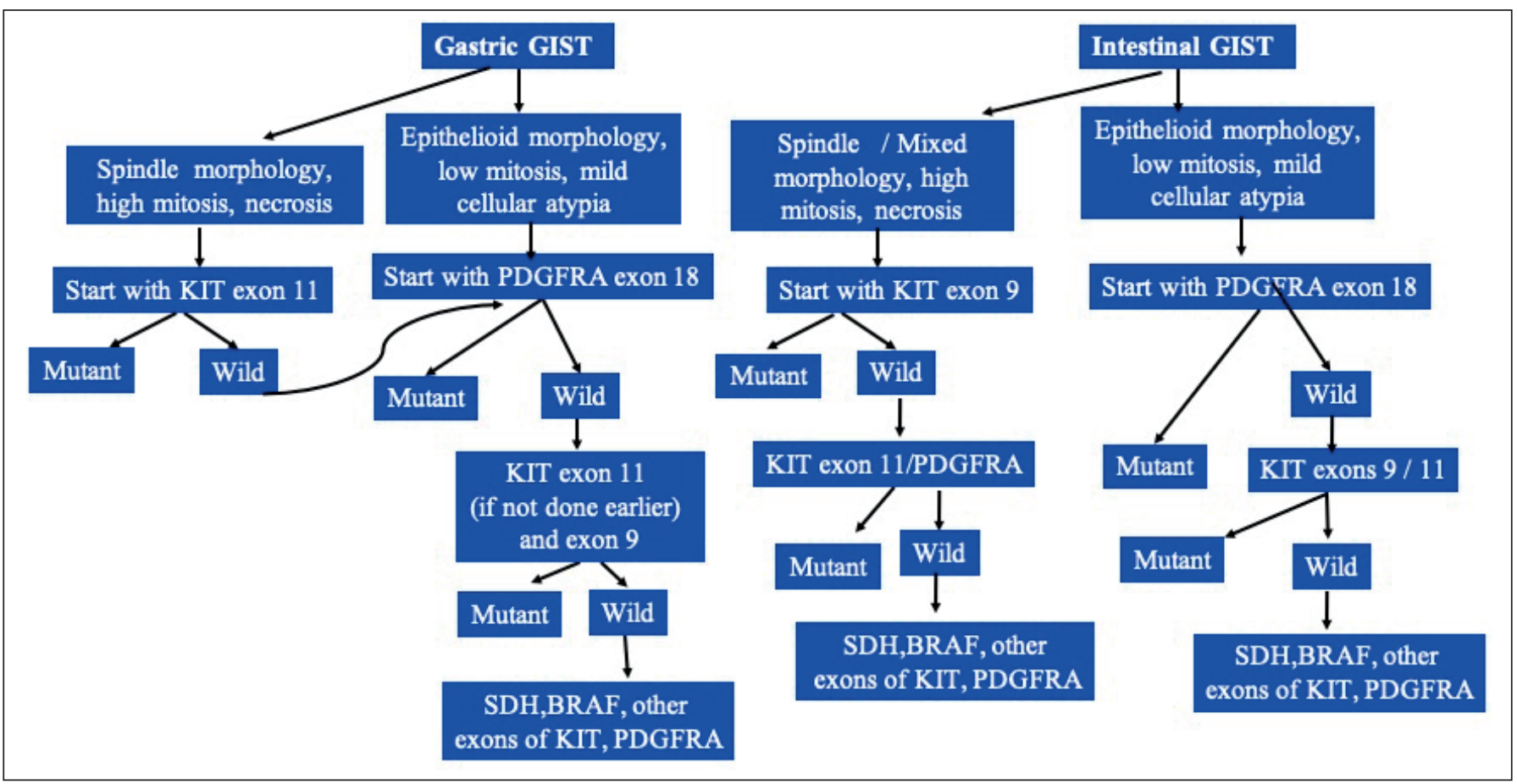

Figure 4: The figure shows suggested algorithm for molecular testing in GIST.

In conclusion, this study is dedicated to the present association of morphological features with mutations occurring in GIST. Genotyping GIST is becoming mandatory as it is recommended to predict dosage and response to imatinib therapy. The only matter of concern is its availability and affordability for the individual patient. Since GISTs harbor multiple mutations, next generation sequencing (NGS) is the most suitable technology today to study mutations. However, the biggest limitations with NGS are the cost and the necessary expertise which is not available in all centres. Therefore, most of the laboratories have to depend on the gold standard Sanger sequencing and it is always prudent to prioritize the mutation testing especially in settings with limited financial means. We suggest testing initially for KIT exon 11 and/or PDGFR exon 18 in gastric GISTs depending on spindle cell and epithelioid or mixed cell morphology respectively, followed by KIT exon 13, 17 and PDGFRA exon12.

\section{CONFLICT of INTEREST}

The authors declare no conflict of interest.

\section{REFERENCES}

1. Wahem A, Schaefer IM, Schüler P, Cameron S, Ghadimi BM. Gastrointestinal stromal tumours. Int J Colorectal Dis. 2012;27:689-700.
2. Hirota S, Isozaki K, Moriyama Y,Hashimoto K, Nishida T, Ishiguro S, Kawano K, Hanada M, Kurata A, Takeda M, Muhammad Tunio G, Matsuzawa Y, Kanakura Y, Shinomura Y, Kitamura Y. Gain-of-function mutations of c-kit in human gastrointestinal stromal tumours. Science. 1998;279:577-80.

3. Corless CL, Schroeder A, Griffith D, Town A, McGreevey L, Harrell P, Shiraga S, Bainbridge T, Morich J, Heinrich MC. PDGFRA mutations in gastrointestinal stromal tumours: Frequency, spectrum and in vitro sensitivity to imatinib. J Clin Oncol. 2005;23:5357-64.

4. Zhao X, Yue C. Gastrointestinal stromal tumours. J Gastrointest Oncol. 2012;3:189-208.

5. Andersson J, Bumming P, Meis-Kindblom JM. Gastrointestinal stromal tumours with KIT exon 11 deletions are associated with poor prognosis. Gastroenterology. 2006;130:1573-81.

6. Lasota J, Kuban W, Wardelmann E, Debiec-Rychter M, Merkelbach-Bruse S, Sciot R, Rys J, Steigen SE, Iwanik K, Holden JA, Jerzak Vel Dobosz A, Schildhaus HU, Miettinen M. KIT codon 558 insertions in gastrointestinal stromal tumors. Analysis of 17 rare KIT mutants. Hum Pathol. 2008;39:1728-36.

7. Daum O, Grossmann P, Vanecek T, Sima R, Mukensnabl P, Michal M. Diagnostic morphological features of PDGFRAmutated gastrointestinal stromal tumours: Molecular genetic and histologic analysis of 60 cases of gastric gastrointestinal stromal tumours. Ann Diagn Pathol. 2007;11:27-33.

8. Miettinen M, Lasota J. Gastrointestinal stromal tumours: Pathology and prognosis at different sites. Semin Diagn Pathol. 2006;23;70-83. 
9. Miettinen M, Sobin LH, Lasota J. Gastrointestinal stromal tumours of the stomach: A clinicopathologic, immunohistochemical, and molecular genetic study of 1765 cases with long-term follow-up. Am J Surg Pathol. 2005;29:52-68.

10. Sandvik OM, Soreide K, Kvaloy JT, Gudlaugsson E, SøreideJ A. Epidemiology of gastrointestinal stromal tumours: Singleinstitution experience and clinical presentation over three decades. Cancer Epidemiol. 2011;35:515-20.

11. Garcés-Albir M, Marti-Obiol R, López-Mozos F, CalabuigFariñas S, Navarro-Ros S, Ortega-Serrano J. Results on prognostic value of mutations in localized gastrointestinal stromal tumours (GIST) in one single center. Rev Esp Enferm Dig. 2012;104:40510.

12. Vij M, Agrawal V, Kumar A, Pandey R. Gastrointestinal stromal tumours: A clinicopathological and immunohistochemical study of 121 cases. Indian J Gastroenterol. 2010;29:231-6.

13. Ravikumar G, Kalegowda IY, Ananthamurthy A: Clinicopathological spectrum of gastrointestinal stromal tumours-experience at a tertiary care centre. Ind J Cancer. 2011;48: 466-470.

14. Kim KM, Kang DW, Moon WS, Park JB, Park CK, Sohn JH, Jeong JS, Cho MY, Jin SY, Choi JS, Kang DY. Gastrointestinal stromal tumors in Koreans: It's incidence and the clinical, pathologic and immunohistochemical findings. J Korean Med Sci. 2005;20:97784.

15. Foo WC, Liegl-Atzwanger B, Lazar AJ. Pathology of gastrointestinal stromal tumors. Clin Med Insights Pathol. 2012;5:23-33.

16. Shi Y, Au JS, Thongprasert S, Srinivasan S, Tsai CM, Khoa MT, Heeroma K, Itoh Y, Cornelio G, Yang PC. A prospective, molecular epidemiology study of EGFR mutations in Asian patients with advanced non-small-cell lung cancer of adenocarcinoma histology (PIONEER). J Thorac Oncol. 2014;9:154-62.

17. Ahmad F, Lad P, Bhatia S, Das BR. Molecular spectrum of c-KIT and PDGFRA gene mutations in gastro intestinal stromal tumour: Determination of frequency, distribution pattern and identification of novel mutations in Indian patients. Med Oncol. 2015;32:424.

18. Cyriac S, Rajendranath R, Sagar TG. Gastrointestinal stromal tumour: Analysis of outcome and correlation with c-kit status in Indian population. Indian J Cancer. 2014;51:35-9.
19. Chiang NJ, Chen LT, Tsai CR, Chang JS. The epidemiology of gastrointestinal stromal tumours in Taiwan, 1998-2008: A nationwide cancer registry-based study. BMC Cancer. 2014;14:102.

20. Wozniak A, Rutkowski P, Piskorz A, Ciwoniuk M, Osuch C, Bylina E, Sygut J, Chosia M, Rys J, Urbanczyk K, Kruszewski W, Sowa P, Siedlecki J, Debiec-Rychter M, Limon J; Polish Clinical GIST Registry. Prognostic value of KIT/PDGFRA mutations in gastrointestinal stromal tumours (GIST): Polish Clinical GIST Registry experience. Ann Oncol. 2012;23:353-60.

21. Katsarou K, Dimitriadis E, Lariou C, Kairi-Vassilatou E, Pandis N, Kondi-Paphiti A. KIT exon 11 codon 557/558 deletion/ insertion mutations define a subset of gastrointestinal stromal tumours with malignant potential. World J Gastroenterol. 2008;14:1891-7.

22. Daniels M, Lurkin I, Pauli R, Erbstösser E, Hildebrandt U, Hellwig K, Zschille U, Lüders P, Krüger G, Knolle J, Stengel B, Prall F, Hertel K, Lobeck H, Popp B, Theissig F, Wünsch P, Zwarthoff E, Agaimy A, Schneider-Stock R. Spectrum of KIT/PDGFRA/BRAF mutations and Phosphatidylinositol-3-Kinase pathway gene alterations in gastrointestinal stromal tumours (GIST). Cancer Lett. 2011;312:43-54.

23. Lv M, Wu C, Zheng Y, Zhao N. Incidence and survival analysis of gastrointestinal stromal 342 tumors in shanghai: A populationbased study from 2001 to 2010. Gastroenterol Res Pract. 2014;2014:834136.

24. Agaimy A, Wunsch PH, Hofstaedter F,Blaszyk H, Rümmele P, Gaumann A, Dietmaier W, Hartmann A. Minute gastric sclerosing stromal tumours (GIST tumourlets) are common in adults and frequently show c-KIT mutations. Am J Surg Pathol. 2007;31:113-20.

25. Boikos SA, Pappo AS, Killian JK, LaQuaglia MP, Weldon CB, George S, Trent JC, von Mehren M, Wright JA, Schiffman JD, Raygada M, Pacak K, Meltzer PS, Miettinen MM, Stratakis C, Janeway KA, Helman LJ. Molecular subtypes of KIT/PDGFRA wild-type gastrointestinal stromal tumours: A report from the National Institutes of Health Gastrointestinal Stromal Tumour Clinic. JAMA Oncol. 2016;2:922-8.

26. Pantaleo MA, Nannini M, Corless CL, Heinrich MC. Quadruple wild-type (WT) GIST: Defining the subset of GIST that lacks abnormalities of KIT, PDGFRA, SDH, or RAS signaling pathways. Cancer Med. 2015;4:101-3. 